\title{
Cardiac perception and the voluntary control of heart rate
}

\author{
LINDA R. GANNON \\ Southern Illinois University, Carbondale, Illinois 62901
}

\begin{abstract}
Twenty-four men and twenty-four women participated in an experiment designed to examine the relationships among cardiac interoception, external feedback, and the voluntary control of heart rate. The concept of cardiac interoception was operationalized as heart-rate estimation in Experiment 1 and as heart-rate discrimination in Experiment 2. The heart-rate estimation task proved to be the more reliable and productive of the two operations in assessing perception of internal cues associated with cardiac activity. The cardiac estimation data suggested that instructional set and type of feedback did influence the learning of cues related to cardiac perception but that feedback did not enable subjects to learn to perceive their cardiac activity accurately. Analysis of the relationship between cardiac awareness and heart-rate control indicated that the facilitating effects of feedback on voluntary control are not mediated by cardiac awareness and that this model of biofeedback is inappropriate.
\end{abstract}

There is reasonable agreement among investigators that external feedback facilitates the voluntary control of heart rate (e.g., Blanchard \& Young, 1973; Lang \& Twentyman, 1974); however, the mechanism by which feedback facilitates voluntary control is not clear. One possible explanation for this phenomenon is that feedback acts to improve interoception or the perception of autonomic cues. This model of visceral learning predicts that: (1) external feedback enhances the accuracy of autonomic perception, and (2) enhanced autonomic perception is associated with increased control of autonomic functions. The present study evaluates this hypothesis, that is, that the modification of visceral responses through feedback is mediated by an enhancement of internal cues.

Previous researchers have investigated the effects of external feedback on cardiac perception. Epstein, Cinciripini, McCoy, and Marshall (1977) and Epstein and Stein (1974) studied the potential of heart-rate changes to serve as discriminative stimuli. Subjects were required to indicate if they thought their heart rates were increasing or decreasing. Ashton, White, and Hodgson (1979) measured cardiac perception utilizing techniques from signal detection theory wherein subjects had to choose which of two time periods contained the shortest interbeat (R-R) interval. The results of all three studies suggested that perfor-

This study is based on a dissertation submitted to the University of Wisconsin in partial fulfillment of the requirements of the $\mathrm{PhD}$ degree under the direction of Peter J. Lang, whose assistance in all phases of this project is gratefully acknowledged. I would also like to thank Michael Falconer and Stephen N. Haynes for their help. This research was supported in part by a grant to Peter Lang from the National Institute of Mental Health (MH 10993). Address requests for reprints to: Linda R. Gannon, Department of Psychology, Southern Illinois University, Carbondale, Illinois 62901. mance feedback facilitated cardiac perception. However, as Ashton et al. (1979) and Epstein et al. (1977) have pointed out, these studies were not designed to indicate which sensory events are actually being perceived or what particular strategies were being utilized. Thus, a major purpose of the first of two experiments described below was to identify which cues, of those available in external feedback, were utilized by subjects to improve cardiac perception.

The relationship between cardiac perception and cardiac control has also received attention in previous research. Brener (1974) compared feedback and nofeedback groups on the perception of heart beats and on the ability to increase and decrease heart rate and found that feedback was associated with significantly better cardiac perception and control. The no-feedback control group, however, were not instructed to attend to their cardiac activity; thus, we do not know if the feedback training actually added anything to an instructional set to perceive heart rate. In addition, the superior cardiac control manifested by the highperception group could be attributed to greater exposure to feedback rather than to increased cardiac perception.

McFarland (1975) investigated the relationship between perception and control in a correlational study. He found a significant positive correlation between interoception and control for increasing, but not for decreasing, heart rate. However, the data reported were atypical, since only 11 of the 21 subjects were able to increase their heart rates and the average heart-rate increase was only 1.2 beats per minute (bpm), which is far below increases reported in other studies (e.g., Lang \& Twentyman, 1974). Thus, the studies by Brener and McFarland do not adequately define the relationship between cardiac perception and control. 
The two experiments in the current study were designed to examine the relationship between cardiac perception and cardiac control. The purpose of Experiment 1 was to investigate the effects of external feedback on heart-rate perception. Heart-rate perception was operationalized as degree of accuracy on a task which required subjects to estimate their $R-R$ intervals. Cardiac perception was measured before and after feedback trials. The feedback and instructions were manipulated in such a way as to provide different information to each of four groups.

In Experiment 2, heart-rate perception was operationalized as performance on a heart-rate discrimination task. Subjects viewed either immediate feedback or feedback delayed for $1 \mathrm{~min}$. The purpose of this experiment was threefold: (1) to determine if subjects could discriminate between an immediate and a "delayed" display of their heart rates and, if so, if this skill could be facilitated by rewarding correct discrimination; (2) to compare this task to the heart-rate estimation task as a method for assessing heart-rate perception; and (3) to measure the effects of the previous training in cardiac estimation on heart-rate discrimination ability.

Both Experiment 1 and Experiment 2 included an assessment of the ability to control heart rate. After training in estimation and discrimination, half of the subjects were instructed to slow their heart rates and half were instructed to speed their heart rates. This design allowed for the opportunity to investigate the possibility that cardiac perception affects speeding and slowing performance differentially. No feedback was presented during heart-rate control tasks in order to avoid confounding the influence of cardiac perception and amount of exposure to feedback.

\section{EXPERIMENT 1}

\section{Method}

\begin{abstract}
Subjects
The subjects were 24 men and 24 women, who received credit in their introductory psychology course at the University of Wisconsin for participating in this experiment. All subjects were screened during the initial interview for physical and psychological disorders, medication, and drug usage. The subjects were fully informed of the procedures prior to participation and were allowed to withdraw at any time without penalty.
\end{abstract}

\section{Apparatus}

Heart rate was continuously recorded on a Beckman Type $\mathbf{R}$ dynograph. Beckman silver-silver chloride miniature electrodes were applied over the anterolateral lower ribs. Analogue EKG signals were channeled through a Tektronix Model RM503 oscilloscope that was modified to detect and trigger on each $R$ wave. The output from the scope was connected to a KW12 clock input on a Digital Equipment Corporation (DEC) PDP-12 computer which utilized a Schmitt-trigger to interrupt the computer. This system allowed R-R intervals to be measured to the nearest 250th of a second.

Cardiac information was provided to subjects on a scope slaved to the PDP-12. The display the subject viewed consisted of a moving horizontal line that originated on the left of the screen, terminated at some point on the screen, and then disappeared. Within microseconds, another moving horizontal line began at the left of the screen. In addition, there was a vertical line or index; the position of the index was controlled by the subject.

\section{Procedure}

Subjects were assigned randomly to one of four experimental groups, with the limitation that each group contain six men and six women. The order of events during the experimental procedure was as follows: (1) heart-rate control-two 3-min trials with a 1-min rest between trials; (2) estimation-one 3-min trial with a 3-min rest before and after the trial; (3) monitor-five 3-min trials with a 1-min rest between each trial; (4) estimation-one 3-min trial with a 3-min rest before and after the trial; and (5) heart-rate controltwo 3-min trials with a 1-min rest between trials. The sessions began by applying electrodes. The experimenter then read instructions while the subject watched a sample of the feedback display he/she would see during the monitor trials. The timing, sequence, and presentation of events during the experiment were controlled by the PDP-12 computer.

Estimation. The purpose of the estimation trials was to assess the subject's ability to estimate heart rate. During the heart-rate estimation tasks, the horizontal line terminated at the vertical index. The subject's task was to estimate the interval between heartbeats by moving the index - to the left for shorter intervals and to the right for longer intervals. A subject received a perfect score if he/she positioned the vertical index so that the moving line terminated with each heartbeat.

Monitor. The purpose of the monitor trials was to provide heartrate feedback to the subject. The display represented heart rate; the source of the display and instructional set were the experimental manipulations. The experimental groups were designed as follows: (1) Coincident-Feedback was immediate, and the instructions directed attention toward internal cues associated with beat-by-beat variations. (2) Respiration-Feedback was immediate, and the subjects were told to pay special attention to the relationship between displayed heart-rate change and their ongoing respiratory activity. (3) Delayed-Feedback was delayed approximately $1 \mathrm{~min}$, and the instructions directed attention toward general characteristics of the display-level and variability. (4) Typical-The line terminations were not determined by the subject's own heart rate but were generated by another person's heart rate which had previously been recorded on tape; the instructions directed attention toward learning what heart rate "looks like" when displayed in this manner. In order to ensure that subjects paid attention to the display, they were required to "track" the display by moving the vertical index to coincide with line terminations. All subjects were informed as to the source of their display and as to whether it was delayed or immediate.

Heart-rate control. Half of the subjects were requested to increase their heart rates for all heart-rate control trials, and half were requested to decrease their heart rates. The subjects were not told beforehand which task they would be asked to do. While feedback was presented during the task, the subject was informed after each trial as to what his/her heart rate (in beats per minute) had been during the control task and how much this differed from his/her rate at rest.

\section{Data Reduction}

For the estimation task, accuracy of cardiac perception was measured by the difference between the index position and the $\mathbf{R}-\mathbf{R}$ interval at the time of a heartbeat; for monitor tasks, it was measured by the difference between the index position and the place of the line termination. ${ }^{1}$ An average error was obtained for each trial. Summary statistics describing central tendency and variability for each cell for heart period and monitor and estimation errors were printed at the end of each session and stored on digital tape. Medians and interquartile ranges, rather than means and 
standard deviations, were usually selected for analysis because of their relative immunity to extreme scores. In addition, interval histograms for heart period were saved on tape, and these histograms were later edited to eliminate artifacts due to faulty triggering.

\section{Results $^{2}$}

Results pertaining to heart-rate control are presented with the results of Experiment 2.

\section{Heart-Rate Estimation and Monitor}

The primary hypothesis tested by analysis of the estimation and monitor data was that beat-by-beat feedback provides specific information of cardiac activity in addition to the general information on rhythm, variability, and level provided by a noncontingent display. Group differences in estimation performance were analyzed to assess this hypothesis.

Average estimation errors are given in Figure 1. Separate bars are given for initial and final estimation trials. Analysis of variance for the initial estimation trial indicated a significant Group effect $[F(3,40)=$ 3.70, $\mathrm{MSe}=1,482]$, and a post hoc Scheffé test showed the Typical group to be significantly inferior in estimation performance when compared with the average of the other groups $[\mathrm{F}(3,40)=3.35]$; other Scheffé tests were not significant. Prior to the first assessment of estimation ability, the treatment of the groups differed in two respects-in instructions and in the demonstration display presented during the instructions. Since the initial group differences were most probably the result of these treatment manipulations, analysis of covariance or difference scores could not be appropriately utilized to eliminate these differences statistically.

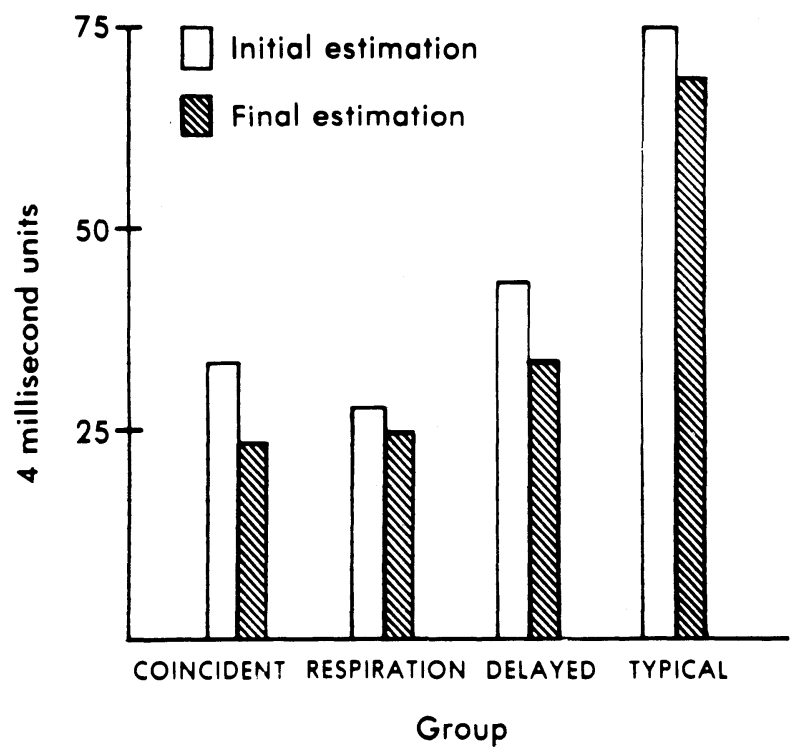

Figure 1. Average estimation errors during initial and final estimation trials for each group $(n=12)$.
Variance analyses of final estimation ability indicated a significant Group effect $[\mathrm{F}(3,40)=5.41$, MSe = 1,036] for the error score. Post hoc Scheffé tests on group means indicated that the Typical group had significantly more errors than the other three groups $[F(3,40)=5.18]$ and that the Coincident and Respiration groups averaged fewer errors than the Typical and Delayed groups $[F(3,40)=2.86]$; other Scheffé tests were not significant. These results suggest that subjects provided immediate feedback were better estimators than those not provided such feedback. However, since there were initial estimation group differences, and since the Coincident group did not differ significantly from either the Respiration or the Delayed groups, it is not clear from these analyses if groups differed in terms of the strategy utilized to estimate heart rate. Thus, further analyses involving correlational techniques were done.

\section{Correlative Relationships}

Analysis of the association between the median estimated heart rate and the actual heart rate yielded significant correlations for the Respiration, Coincident, and Delayed groups but not for the Typical group (see Table 1); this was true for both estimation trials. These variables are more associated with heart-rate level than with beat-by-beat variations, and these correlations may reflect the fact that the Coincident, Respiration, and Delayed groups received information on their rate levels while the Typical group did not.

Additional correlation coefficients were obtained between final estimation error and the difference between the median heart rate monitored (averaged across five trials) and the median heart rate during the final estimation. It is suggested that if, during the final estimation trial, subjects were simply mimicking what they had monitored, then greater estimation error would have been associated with greater differences between heart rate during monitoring and heart rate during estimating. That is, if, when estimating, subjects simply reproduced a display similar to their monitor display, then those subjects whose actual heart-rate characteristics changed from monitoring to estimating would be rated as poor estimators. The correlation coefficients suggest that the mimic effect is indicated for the Delayed group $(r=.687)$. However, these correlations were insignificant for the Coincident $(r=.472)$ and Respiration $(r=.327)$ groups, suggesting that these groups relied to a lesser extent on mimicking the monitor display.

Finally, correlations between the variability of estimated heart rate and the variability of actual heart rate yielded a significant correlation for the Delayed group during final estimation $(r=.620)$; the other correlations did not reach significance. These data suggest that the Delayed groups followed instructions and attended to variability characteristics and 
Table 1

Correlations Between Median Actual Heart Rate and Median Estimated Heart Rate for Initial and Final Estimation Trials

\begin{tabular}{ccccc}
\hline & \multicolumn{4}{c}{ Actual vs. Estimated } \\
\cline { 2 - 5 } Estimation & COIN & RESP & DEL & TYP \\
\hline Initial & $.820^{*}$ & $.842^{*}$ & $.687^{*}$ & .326 \\
Final & $.734^{*}$ & $.634^{*}$ & $.686^{*}$ & .304 \\
\hline
\end{tabular}

Note $-n=12 . \quad{ }^{*} p<.05$.

that they used this information when estimating their heart rate.

To summarize briefly, although the analysis of the error scores for the final estimation trial yielded a significant Group effect and although the group means were distributed in the predicted manner, the Coincident, Respiration, and Delayed groups were not significantly different from one another. Within-group correlation coefficients suggested that the Coincident, Respiration, and Delayed groups utilized level information; and, although the Coincident and Respiration groups were better (although not significantly so) at estimating heart rate than was the Delayed group, the last group apparently estimated by mimicking the monitor display and/or learning variability patterns, whereas the former groups did not.

\section{EXPERIMENT 2}

\section{Method}

\section{Subjects}

Twenty-four subjects from Experiment 1, six from each of the four groups, were selected to participate in Experiment 2.

\section{Apparatus}

The apparatus was identical to that of Experiment 1 except that the index on the sweep display was centered and stationary.

\section{Procedure}

The subjects were assigned to either the experimental group or the control group so that the design was completely crossed and balanced for sex, control task (speeding/slowing), and group from Experiment 1.

The session began with all subjects performing the discrimination task. The subjects then received training in discrimination and, finally, were reassessed on the discrimination task. The session ended with all subjects' participating in a heart-rate control segment identical to that in Experiment 1.

Discrimination task. The discrimination task consisted of 10 trials. For each trial; the subject observed a display, generated either by immediate heart rate or by delayed heart rate, in which the length of the moving horizontal line was proportional to the $\mathbf{R}-\mathbf{R}$ interval. After $30 \mathrm{sec}$, the subject was required to decide if the display had been generated by immediate or by delayed heart rate by moving an arrow to one side of the screen or the other. The subject had as much time as necessary to make the choice and, after choosing, pressed a button to continue. After 10 trials, the subject was told how many trials had been correct.

Training. The training trials for the experimental group were identical to the test trials, except that after each decision the subject was informed as to the correctness of the decision and there was no scoreboard at the end of a set of trials. The training trials for the control group were not associated with the subject's heart rate. The displays were computer generated, and the subject's task was to decide, after a 30 -sec display, whether more lines had terminated on the left or more lines had terminated on the right of the vertical center line. Instructions to subjects indicated that this training would increase their ability to concentrate on the display and, thus, improve their heart-rate discrimination ability. As with the experimental group, the control group subjects were informed, after each trial, of the correctness of their decisions. There were 1830 -sec trials.

\section{Data Reduction}

Heart and respiration data were processed in the same manner as in Experiment 1.

\section{Results}

\section{Heart-Rate Discrimination}

Due to the more "ordinal" than "interval" nature of the performance data from the discrimination task, nonparametric statistics were used in these analyses. The two groups did not differ on the number correct during the first and second discrimination tasks or on improvement from the first to the second, and neither group showed significant improvement across test trials or training trials.

It was possible to assess the effects on discrimination performance carried over from the estimation training of the first experiment because six subjects from each original group took part in Experiment 2. A KruskalWallis analysis of variance by ranks was carried out for the number of correct trials during each discrimination task. There was a significant Group effect for both tasks $(H=14.75, H=8.03)$; the Respiration group was clearly superior to the other three groups in discrimination ability.

In interpreting these results, it should be noted that, prior to training, a third of the subjects were performing significantly better than chance. Furthermore, the experimenter observed that frequently subjects appeared to be basing their discrimination on whether or not a particular voluntary act (cough, yawn, quick movement) was accompanied by the unconditioned cardiac effect. And, finally, the Respiration group's clear superiority on this task demonstrates the ease with which subjects utilize respiratory cues to perform the task. Given this information, it seems clear that the discrimination task used in the present study does not assess cardiac perception accurately. However, the results presented here do address the question of which task-estimation or discrimination-is the preferred one for assessing perception and provide further differentiation among the groups in Experiment 1.

\section{Heart-Rate Control: Experiments 1 and 2}

Heart-rate performance scores. Performance scores were computed by subtracting the median R-R interval length of the precontrol base from the median interval 
length of each control trial and then changing the algebraic sign of these deviation scores to produce a positive number if the response was in the instructed direction and a negative number if the response was opposite to the instructed direction. These scores enabled evaluation of differences in speeding and slowing ability. The analysis of Experiment 1 heart-rate control data included 48 subjects and yielded a significant Task effect $[\mathrm{F}(1,32)=4.81, \mathrm{MSe}=12,857]$, which suggested that subjects were better able to increase than to decrease their heart rates. Similarly, a significant Task effect $[\mathrm{F}(1,6)=11.19$, MSe $=3,710]$ was found for the heart-rate control data from Experiment 2 . The magnitudes of the heart-rate changes were consistent with those found in other studies employing similar methodologies (Gatchel, 1974; Lang \& Twentyman, 1974). The average change during the best trial for speeding was $104 \mathrm{msec}$ and for slowing was $43 \mathrm{msec}$ (assuming a base rate of $70 \mathrm{bpm}$, these times translate to approximately 10 and $3.5 \mathrm{bpm}$, respectively).

It was hypothesized that interoceptive ability, evidenced by either estimation performance or discrimination performance, would facilitate instructional control of heart rate. Thus, it was expected that the Coincidence, Respiration, Delayed, and Typical groups would differ in ability to increase and decrease heart rate. There was, however, not a significant Group effect for the Experiment 1 heart-rate control data. It was also expected that, in Experiment 2, those subjects given heart-rate discrimination training would be superior in heart-rate control to those subjects given "attention" training. This hypothesis was also not supported in that there was not a significant Group effect for the Experiment 2 heart-rate control data.

\section{Correlative Relationships}

Each group was divided according to speeding and slowing subjects for the purpose of this analysis. Although this resulted in a reduced number of degrees of freedom associated with these correlations, it was considered necessary in order to investigate the possibility of differential effects of cardiac perception on speeding and slowing performance. A correlation matrix that included the following variables was obtained: the final estimation error score, the percent improvement in estimation ability from the first to the second trial, the preestimation control performance, and the pre- to postestimation improvement in control performance. Of the 32 correlation coefficients, only one was significant in the predicted direction. ${ }^{3}$ There were no significant correlations associating heart-rate discrimination performance with heart-rate control performance.

\section{DISCUSSION}

The primary purpose of the present study was to evaluate the hypothesis that the modification of visceral responses with the aid of feedback is mediated by an improved perception of internal cues. The results of the present study indicate that this is not an appropriate model with which to define feedback-produced instructional control of heart rate. Although three of the four groups received feedback related to their own heart rates and these groups were significantly better at perceiving their cardiac activity than was the group whose feedback was not related to heart rate, these differences in perception were not paralleled by group differences in ability to speed or slow heart rate. Furthermore, regardless of feedback training, one might hypothesize that certain individuals might be more perceptive to internal cues than others and that these individuals might excel in controlling their heart rates. However, the relationship between perception and control was not significant. Thus, these results suggest that feedback does not aid control by improving the perception of internal cues.

The validity of this conclusion is clearly dependent upon our confidence that the tasks were adequate measures of cardiac perception. Two perception tasks were used in the present study. The estimation task was clearly superior to the discrimination task in that success on the latter was too easily achieved by the use of skeletal manipulations. On the other hand, one could argue that, since errors in estimation were scored on a beat-by-beat basis, the estimation task does not represent a realistic demand; that is, the subject is assumed to perceive an interoceptive cue and to react to it in a period of time less than the R-R interval. However, this method of scoring was highly correlated with an error score reflecting the difference between estimated and actual median R-R intervals, and neither score correlated with heart-rate control. Given the absence of a criterion for determining the validity of a perception measure, it cannot be concluded that these are the best measures of perception. However, the present study represents the most comprehensive evaluation of cardiac perception to date, and none of the measures of cardiac perception were related to ability to modify heart rate.

Although the lack of a relationship between cardiac perception and cardiac control is inconsistent with the model being tested, of secondary interest is the effect of feedback on perception. The primary test of this was the comparison between the Coincident and Delayed groups which, although in the predicted direction, was not significant. This suggests that persons do not learn anything unique about their cardiac activity by viewing an immediate, beat-by-beat display of their heart rates. The correlation analyses suggest that the various groups learned different strategies and/or to recognize different cues, but this differential learning was not reflected in differences on the perception task. Indeed, the results lead one to question whether perception of those interoceptive cues 
related to cardiac activity is possible. The Coincident group was instructed to attend to internal cues, carefully attended to $15 \mathrm{~min}$ of beat-by-beat feedback, and performed the best when tested on their perception. Yet even this group's average error was approximately $100 \mathrm{msec}$, which, assuming a basal heart rate of $60 \mathrm{bpm}$, transforms to $10 \mathrm{bpm}$. More intensive training and different tasks may, of course, yield different results, but the data from the present study indicate that subjects did not learn to perceive their cardiac activity accurately.

In conclusion, the theoretical model of trained interoception is an inappropriate one with which to define and study feedback-produced instructional control of heart rate. Other models of biofeedback such as "instrumental conditioning of autonomic responses" (Miller, 1969) and "trained exteroception" (Brener, 1974) remain to be evaluated.

\section{REFERENCES}

Ashton, R., White, K. D., \& Hodgson, G. Sensitivity to heart rate: A psychophysical study. Psychophysiology, 1979, 16, 463-466.

Blanchard, E. G., \& Young, L. D. Self-control of cardiac functioning: A promise yet unfulfilled. Psychological Bulletin, 1973, 79, 145-163.

Brener, J. A general model of voluntary control applied to the phenomena of learned cardiovascular change. In P. A. Obrist, A. H. Black, J. Brener, \& L. V. DiCara (Eds.), Cardiovascular psychophysiology. Chicago: Aldine, 1974.

Epste in, L. H., Cincirpini, P. N., McCoy, J. F., \& Marshall, W. R. Heart rate as a discriminative stimulus. Psychophysiology, 1977, 14, 143-149.
Epste in, L. H., \& Stein, D. Feedback-influenced heart rate discrimination. Journal of Abnormal Psychology, 1974, 83, 585-588.

Gatches, R. J. Frequency of feedback and learned heart rate control. Journal of Experimental Psychology, 1974, 103, 274-283.

Lang, P. J., \& Twentyman, C. T. Learning to control heart rate: Binary versus analogue feedback. Psychophysiology, 1974, 11, 616-629.

MCFARLAND, R. A. Heart rate perception and heart rate control. Psychophysiology, 1975, 12, 402-405.

Mille R, N. E. Learning of visceral and glandular responses. Science, 1969, 163, 434-445.

\section{NOTES}

1. A second measure of estimation error was computed. This was the difference between the median actual $R-R$ interval and the median estimated R-R interval. Since the two were highly correlated $(r=.86$ and $r=.95)$ and yielded similar results, only the first is reported.

2. The .05 rejection level was adopted in all statistical tests unless otherwise indicated.

3. The Respiration group showed a consistent pattern (three out of four) of significant correlations relating good estimating performance with poor slowing performance. Apparently, those subjects who were superior in learning and/or attending to phasic heart-rate changes associated with respiratory activity were inferior in their ability to decrease their heart rate. This effect was possibly due to these subjects' having their attention directed toward respiratory activity rather than cardiac activity. Although these data are only suggestive, the practice of requiring subjects to match their respiration to a simulator to prevent respiratory mediation of heart-rate changes could hinder slowing performance.

(Received for publication February 5, 1980; revision accepted June 19,1980 .) 